\title{
ON SINGULAR PROJECTIVE DEFORMATIONS OF TWO SECOND CLASS TOTALLY FOCAL PSEUDOCONGRUENCES OF PLANES
}

\section{LUDMILA GOLDBERG}

Department of Mathematics

New Jersey Institute of Technology

Newark, New Jersey 07102 U.S.A.

(Recieved Ju1y 31, 1986)

ABSTRACT. Let $C: L \rightarrow \bar{L}$ be a projective deformation of the second order of two totally focal pseudocongruences $L$ and $\bar{L}$ of $(m-1)$-planes in profective spaces $P^{n}$ and $\bar{P}^{n}$, $2 m-1 \leq n<3 m-1$, and let $K$ be a collineation realizing such a $C$. The deformation $C$ is said to be weakly singular, singular, or $\alpha$-strongly singular, $\alpha=3,4, \ldots$, if the collineation $\mathrm{K}$ gives projective deformations of order 1,2 or $\alpha$ of all corresponding focal surfaces of $\mathrm{L}$ and $\overline{\mathrm{L}}$. It is proved that $\mathrm{C}$ is weakly singular and conditions are found for $\mathrm{C}$ to be singular. The pseudocongruences $\mathrm{L}$ and $\overline{\mathrm{L}}$ are identical if and only if $C$ is 3 -strongly singular.

KEY WORDS AND PHRASES. Pseudocongruence, projective deformation, singular projective deformation, focal surface. 1980 MATHEMATICS SUBJECT CLASSIFICATION CODE. 53A20

\section{INTRODUCTION.}

Let $L$ and $\bar{L}$ be totally focal pseudocongruences of (m-1)-planes in projective spaces $\mathrm{P}^{\mathrm{n}}$ and $\overline{\mathrm{P}}^{\mathrm{n}}$ and let $\mathrm{C}: \mathrm{L} \rightarrow \overline{\mathrm{L}}$ be a correspondence between planes of $\mathrm{L}$ and $\overline{\mathrm{L}}$. In the case of pseudocongruences of straight lines $(m=2)$ regular and singular projective deformations $C$ were studied in many papers (see Svec [1] where one can find further references)。

In the present paper we will suppose that $m>2$ and $2 m-1 \leq n<3 m-1$. The last restriction means that $\mathrm{L}$ and $\overline{\mathrm{L}}$ are of second class, i.e. lie in their second osculating spaces provided that their first osculating spaces are tangent spaces.

The author (see Goldberg [2]) found necessary and sufficient conditions for $C$ to be a projective deformation of order 1,2, and 3. However, conditions under which the pseudocongruences $\mathrm{L}$ and $\overline{\mathrm{L}}$ are identical were not found in [2].

In the present paper we will indicate such a condition in terms of singular projective deformations. Note that second and third order singular projective deformations were studied by the author for every $n \geq 3 \mathrm{~m}-1$ (see Goldberg [3]) and for every $\mathrm{n} \geq 4 \mathrm{~m}-1$ (see Goldberg [4]). Note also that second order singular projective deformations in odd-dimensional projective spaces were considered by Kreizlik $[5,6]$.

If $\mathrm{K}$ is a collineation realizing a projective deformation $C$ of second order, and at the same time $K$ realizes projective deformations of order 1,2 , or $\alpha, \alpha=3,4, \ldots$, of all corresponding focal surfaces of $L$ and $\bar{L}$, then $C$ is called weakly singular, 
singular, or $\alpha$-strongly singular respectively.

In the present paper it is proved that a second order projective deformation $C$ is weakly singular, necessary and sufficient conditions for $C$ are found to be singular, and the following condition of identity of $\mathrm{L}$ and $\overline{\mathrm{L}}$ is obtained: pseudocongruences $\mathrm{L}$ and $\overline{\mathrm{L}}$ related by a second order projective deformation $\mathrm{C}: \mathrm{L} \rightarrow \overline{\mathrm{L}}$ are identical if and only if $C$ is 3 -strongly singular.

Note that the author proved in [2] that if $L \subset P^{n}$ and $C: L \rightarrow \bar{L}$ is a projective deformation, then $\overline{\mathrm{L}} \subset \overline{\mathrm{P}}^{\mathrm{n}}$. Because of this, we suppose from the beginning that $\mathrm{L} \subset \mathrm{P}^{\mathrm{n}}$ and $\overline{\mathrm{L}} \subset \overline{\mathrm{P}}^{\mathrm{n}}$.

2. A SPECIALIZATION OF MOVING FRAMES ASSOCIATED WITH A TOTALLY FOCAL PSEUDOCONGRUENCE AND FUNDAMENTAL EQUATIONS.

A family $L$ of planes of an $n$-dimensional projective space $P^{n}$ is said to be a pseudocongruence if each hyperplane of $\mathrm{P}^{\mathrm{n}}$ contains 1ocally a unique plane of $\mathrm{L}$ 。

A pseudocongruence $L$ of $(m-1)$-planes is a family of $m$ parameters. The admissible m-tuples $\left(u_{1}, \ldots, u_{m}\right)$ are taken from an open neighborhood of $C^{m}(C=$ complex numbers $)$.

A one-parameter subfamily of $L$ is said to be focal of order $r$ if infinitesimally close planes of $\mathrm{L}$ have an $\mathrm{r}$-dimensional intersection. Focal subfamilies of maximum order $\mathrm{m}-2$ are called developable surfaces of L. A pseudocongruence of (m-1)-planes possessing the maximum number $m$ of developable surfaces is called focal.

In general, (m-2)-dimensional characteristics of each of these $m$ developable surfaces forms a symplex in a plane $p_{m-1} \in L$. The vertices of this symplex are foci of $p_{m-1}$. Each focus generates the focal surface of $L$ of dimension $m_{0}$ A plane $p_{m-1}$ belongs to the tangent $\mathrm{m}-\mathrm{plane}$ of each of the $\mathrm{m}$ focal surfaces.

It was shown by Geidelman [7] that focal pseudocongruences can be of three types:

(a) Pseudocongruences whose $(m-1)$-planes belong to an $(m+1)-p l a n e$;

(b) Pseudocongruences foliating into $\infty^{b}$ subfamflies of $m-b$ parameters, $1 \leq b \leq m$, where all (m-1)-planes of each of these subfamilies belong to an $m-p l a n e ;$

(c) Pseudocongruences possessing $m$ systems of integrable ( $m-1)$-parameter focal subfamilies of order zero.

Pseudocongruences of the third type are called totally focal (abbreviated t.f.). Each of the $m$ focal surfaces of a t.f. pseudocongruences is an m-conjugate system (see Geidelman [7]).

Let $L$ be a t.f. pseudocongruence of $(m-1)$-planes $p_{m-1}$ in $p^{n}$. To each plane $\mathrm{P}_{\mathrm{m}-1} \in \mathrm{L}$ we associate a moving frame consisting of linearly independent analytic points $A_{1}, \ldots, A_{n+1}$, such that

$$
\left[A_{1}, \ldots, A_{n+1}\right]=1
$$

and $\left(A_{1}, \ldots, A_{m}\right)=p_{m-1}$.

The equations of infinitesimal displacements of the moving frame are

$$
\mathrm{dA}_{\mathrm{u}}=\omega_{\mathrm{u}}^{\mathrm{v}_{\mathrm{A}}}, \quad \mathrm{u}, \mathrm{v}=1, \ldots, \mathrm{n}+1,
$$

where the Pfaffian forms $\omega_{u}^{v}$ satisfy the structure equations (i.e. the integrability conditions) of the space $\mathrm{P}^{\mathrm{n}}$ :

$$
\mathrm{d} \omega_{\mathrm{u}}^{\mathrm{v}}=\omega_{\mathrm{u}}^{\mathrm{w}} \wedge \omega_{\mathrm{w}}^{\mathrm{v}}, \quad \mathrm{u}, \mathrm{v}, \mathrm{w}=1, \ldots, \mathrm{n}+1 .
$$

In addition, differentiating $(2.1)$ by means of $(2.2)$, we obtain

$$
\omega_{u}^{u}=0 \text {. }
$$


In this paper we will suppose that $2 m-1 \leq n<3 m-1$. In this case we can specialize the moving frames in such a way that

(i) the vertices $A_{1}, i=1, \ldots, m$, are foci of $p_{m-1}$;

(ii) the line $A_{i} A_{m+i}$ is tangent to the line $\gamma_{i}$ of the conjugate net ( $A_{i}$ ) which is not tangent to $\mathrm{P}_{\mathrm{m}-1}$;

(iii) the points $A_{2 m+1}, \ldots, A_{2 m+\sigma}$, where $2 m+\sigma=n+1$, are chosen arbitrarily (of course, (2.1) is supposed to be satisfied).

Under such a choice of vertices $A_{u}$ of the moving frames the developable surfaces of $\mathrm{L}$ are determined by equations $\omega_{i}^{m+i}=0$. Since all foci are supposed to be 1 inearly independent, forms $\omega_{i}^{\mathrm{m}+i}$ are also linearly independent. We will take them as forms of the dual cobasis and will denote them by $\omega^{i}$ :

$$
\omega_{i}^{m+i}=\omega^{i} \text {. }
$$

In (2.5) and in what follows there is no summation of the indices $1, j, k=1, \ldots, m$, unless it is indicated by the summation sign. If the moving frames are specialized in the above described manner, we have:

$$
\begin{gathered}
\mathrm{dA}_{i}=\omega_{i}^{k} A_{k}+\omega^{i} A_{m+1}, \\
\omega_{i}^{m+j}=0, \quad j \neq i, \\
\omega_{i}^{2 m+r}=0, \quad r=1, \ldots, \sigma .
\end{gathered}
$$

In addition, since

$$
\left(d A_{i}, A_{i}, A_{m+i}\right) \equiv 0 \bmod \omega^{j}, \quad j \neq i,
$$

by means of $(2.6)$ we obtain

$$
\omega_{i}^{j}=b_{i}^{j} \omega^{j}, \quad j \neq i
$$

Exterior differentiation of (2.7) and (2.8) by means of (2.2) and (2.10) and application of Cartan's lemma leads to

$$
\begin{aligned}
\omega_{m+i}^{m+j}=c_{i}^{j} \omega^{i}, & j \neq 1, \\
\omega_{m+1}^{2 m+r}=a_{i}^{2 m+r}{ }_{\omega}^{i}, & r=1, \ldots, \sigma .
\end{aligned}
$$

It follows from $(2.1)$ and $(2.12)$ that

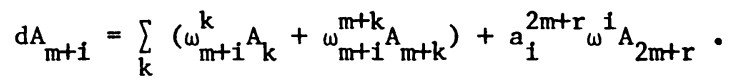

Since $n=2 m-1+\sigma$, there are $\sigma$ linearly independent points among points $a_{1}^{2 m+r} A_{2 m+r}$. Because of this, we have

$$
\operatorname{rank}\left(a_{i}^{2 m+r}\right)=\sigma
$$

In other words, (2.14) means that the second osculating space of $L$ is the whole space $\mathrm{P}^{\mathrm{n}}$, i.e. L is of second class.

Further exterior differentiation of $(2.10),(2.11)$, and (2.12) and application of Cartan's lemma give rise to the following Pfaffian equations:

$$
\begin{gathered}
d b_{i}^{j}+b_{i}^{j}\left(2 \omega_{j}^{j}-\omega_{i}^{i}-\omega_{m+j}^{m+j}\right)-\sum_{i j, j} b_{i}^{k} b_{k}^{j} \omega^{k} \\
=b_{i j}^{j} \omega^{j}+a_{i j}^{j} \omega^{i}, \\
\omega_{m+i}^{j}=a_{i j}^{j} \omega^{j}-a_{i j}^{j} \omega^{i},
\end{gathered}
$$




$$
\begin{gathered}
d c_{i}^{j}+c_{1}^{j}\left(\omega_{1}^{1}-2 \omega_{m+1}^{m+1}+w_{m+j}^{m+j}\right)+\sum_{k \neq 1, j} c_{1}^{k} c_{k}^{j} \omega^{k}+a_{1}^{2 m+s} w_{2 m+s}^{m+j}=a_{11}^{j} \omega^{j}+c_{11}^{j} \omega^{1}, \\
d a_{i}^{2 m+r}+a_{i}^{2 m+r}\left(w_{i}^{1}-2 \omega_{m+1}^{m+1}+w_{2 m+r}^{2 m+r}\right)+a_{1}^{2 m+s} w_{2 m+s}^{2 m+r}+\sum_{k \neq 1} c_{1}^{k} a_{k}^{2 m+r} \omega^{k}=a_{11}^{2 m+r} \omega^{1} .
\end{gathered}
$$

In the following we will need the differential extensions of (2.15) and (2.16) which have the following form:

$$
\begin{aligned}
& d b_{1 j}^{j}+b_{1 j}^{j}\left(3 \omega_{j}^{j}-\omega_{1}^{1}-2 \omega_{m+j}^{m+j}\right)+b_{1}^{j}\left(3 \omega_{m+j}^{j}-a_{j}^{2 m+r} \omega_{2 m+r}^{m+j}\right)-\sum_{k \neq 1, j} b_{1}^{k} b_{k j}^{j} \omega^{k}-3 b_{j}^{1}\left(b_{1}^{j}\right)^{2} \omega^{1} \\
& +3 b_{i}^{j} \sum_{k \neq 1, j} b_{j}^{k} b_{k}^{j} \omega^{k}-b_{i}^{j} \sum_{k \neq 1, j} c_{j}^{k} c_{k}^{j} \omega^{k}-b_{i}^{j} c_{j}^{1} c_{i}^{j} \omega^{1}=b_{i j j}^{j} \omega^{j}+a_{i j j}^{j} \omega^{1}, \\
& d a_{j j}^{j}+a_{i j}^{j}\left(2 \omega_{j}^{j}-\omega_{m+1}^{m+1}-\omega_{m+j}^{m+j}\right)-b_{1}^{j} \omega_{m+1}^{1}+\sum_{k \neq 1, j}\left(a_{11}^{k} b_{k}^{j}-c_{1}^{k} a_{k j}^{j}\right) \omega^{1}-\sum_{k \neq 1, j} b_{k}^{j} a_{1 k}^{k} w^{k} \\
& =a_{i j j}^{1} \omega^{j}-a_{11 j}^{j} \omega^{1} \text {, } \\
& d a_{i 1}^{j}+a_{i 1}^{j}\left(\omega_{i}^{1}-2 \omega_{m+1}^{m+1}+\omega_{j}^{j}\right)-c_{1}^{j} \omega_{m+j}^{j}+\sum_{k \neq 1, j} c_{1}^{k} a_{k k}^{j} \omega^{k}-a_{1}^{2 m+r} \omega_{2 m+r}^{j} \\
& =a_{i 1 j}^{j} \omega^{j}-c_{i 1 j}^{j} \omega^{1} \text {. }
\end{aligned}
$$

3. FIRST AND SECOND ORDER PROJECTIVE DEFORMATIONS OF T.F. PSEUDOCONGRUENCES.

It is well known that $(m-1)$-planes $p_{m-1}$ of the space $P^{n}$ can be represented as points of the Grassmannian $G(m-1, n)$, dim $G=m(n-m+1)$, in a projective space $g\left(P^{n}\right)=P^{N}$ of dimension $N=\left(\begin{array}{c}n+1 \\ m\end{array}\right)-1$. Denote by $\left[M_{1}, \ldots, M_{m}\right]$ Grassmann coordinates of the plane $\left(M_{1}, \ldots, M_{m}\right)$. If $\left\{A_{u}\right\}$ is a moving frame in $P^{n}$, then $\left\{\left[A_{u_{1}}, \ldots, A_{u_{m}}\right\}\right\}$ is a moving frame
of $P^{N}$.

Let $\mathrm{P}^{\mathrm{n}}$ and $\overrightarrow{\mathrm{P}}^{\mathrm{n}}$ be two $\mathrm{n}$-dimensional projective spaces with moving frames $\left\{\mathrm{A}_{\mathbf{u}}\right\}$ and $\left\{\bar{A}_{u}\right\}$ and $K: P^{n} \rightarrow \bar{P}^{n}$ be a collineation given by

$$
\mathrm{KA}_{\mathrm{u}}=\alpha_{\mathrm{u}_{\mathrm{v}}^{\mathrm{A}}}^{\mathrm{A}}, \quad \operatorname{det}\left(\alpha_{\mathrm{u}}^{\mathrm{v}}\right) \neq 0 .
$$

The collineation $K$ induces the collineation $g(K): g\left(P^{n}\right) \rightarrow g\left(\bar{P}^{n}\right)$ given by

$$
K\left[A_{u_{1}}, \ldots, A_{u_{m}}\right]=\alpha_{u_{1}}^{v_{1}} \ldots \alpha_{u_{m}}^{v_{m}}\left[\bar{A}_{v_{1}} \ldots \dot{A}_{v_{m}}\right] \text {. }
$$

$\Lambda$ pseudocongruence $L$ is represented in $g\left(P^{n}\right)$ by some surface belonging to $G(m-1, n)$. We will denote 1 t also by $L$.

A correspondence $C: L \rightarrow \bar{L}$ between two t.f. pseudocongruences $L$ and $\bar{L}$ of $P^{n}$ and $\overline{\mathrm{P}}^{\mathrm{n}}$ is said to be a projective deformation of order $h$ if for any plane $\mathrm{p}_{\mathrm{m}-1} \in \mathrm{L}$ there exists a collineation $K: P^{n} \rightarrow \bar{P}^{n}$ such that surfaces $g(K) g(L)$ and $g(\bar{L})$ have the analytic contact of order $h$ at the point $g\left(p_{m-1}\right)$, 1.e. if

$$
\operatorname{Kd}^{S}\left[A_{1} \ldots A_{m}\right]=\sum_{\ell=0}^{S}\left(\begin{array}{c}
h \\
\ell
\end{array} \theta_{\ell} d^{h-\ell}\left[\bar{A}_{1} \ldots \bar{A}_{m}\right]\right.
$$

where $s=0,1, \ldots, h$ and $\theta_{\ell}$ are $\ell$-forms.

Suppose that the moving frames $\left\{\bar{A}_{u}\right\}$ associated to the planes $\bar{p}_{m-1} \in \bar{L}$ are spec1alized similarly to the moving frames associated to the planes $p_{m-1} \in L$. We w111 denote all expressions connected with $\overline{\mathrm{L}}$ by suppressing the overbar. Then we have equations $\overline{(2.1)}-(\overline{2.21})$ if $2 \mathrm{~m}-1 \leq \mathrm{n}<3 \mathrm{~m}-1$.

According to (3.3), the correspondence $C: L \rightarrow \bar{L}$ is a projective deformation of order one if for any $p_{m-1} \in L$ there exists a collineation $K: P^{n} \rightarrow \vec{p}^{n}$ such that 


$$
\begin{aligned}
& K\left[A_{1} \ldots A_{m}\right]=\left[\bar{A}_{1} \ldots \bar{A}_{m}\right], \\
& K d\left[A_{1} \ldots A_{m}\right]=d\left[\bar{A}_{1} \ldots \bar{A}_{m}\right]+\theta_{1}\left[\bar{A}_{1} \ldots \bar{A}_{m}\right]
\end{aligned}
$$

In what follows we will denote the Grassmann products $\left[A_{1} \ldots A_{m}\right],\left[A_{1} \ldots A_{k-1} A_{u_{k}} A_{k+1} \ldots A_{m}\right]$,

$$
\left.\begin{array}{c}
{\left[A_{1} \ldots A_{k-1} A_{u_{k} A_{k+1}} \ldots A_{\ell-1} A_{u_{\ell}}^{A}{ }_{\ell+1} \ldots A_{m}\right], \ldots \text { by }[A],\left[A_{u_{k}}^{k}\right],\left[A_{u_{k}, u_{\ell}}^{k}, \ldots A_{m}\right]=[A], \quad\left[A_{1} \ldots A_{k-1} A_{u_{k}} A_{k+1} \ldots A_{m}\right]=\left[A_{u_{k}}^{k}\right],} \\
{\left[A_{1} \ldots A_{m}\right]} \\
{\left[A_{1} \ldots A_{k-1} A_{u_{k}} A_{k+1} \ldots A_{\ell-1} A_{u_{\ell}} A_{\ell+1} \cdots A_{m}\right]=\left[A_{u_{k}, u_{\ell}}^{k}, \ldots\right.}
\end{array}\right\}
$$

Using notations (3.5), we can write (3.4) in the form

$$
K[A]=[\bar{A}], \quad K d[A]=d[\bar{A}]+\theta_{1}[\bar{A}] .
$$

By means of $(2.1),(2.3)$, and $(2.4)$ one obtains

$$
d[A]=\sum_{i} \omega_{i}^{i}[A]+\sum_{i} \omega^{i}\left[A_{m+i}^{i}\right] .
$$

The author proved in [4] the following theorem:

THEOREM 1。 A correspondence $C: L \rightarrow \bar{L}$ is a projective deformation of first order if and only if $\mathrm{C}$ is developable (i.e. developable surfaces of $\mathrm{L}$ and $\overline{\mathrm{L}}$ correspond to each other under $\mathrm{C}$ ). A collineation $\mathrm{K}$ realizing such a deformation is determined by

$$
\left.\begin{array}{ll}
\mathrm{KA}_{1}=\rho_{i} \overline{\mathrm{A}}_{i}, & \mathrm{KA}_{\mathrm{m}+\mathrm{i}}=\rho_{i} \overline{\mathrm{A}}_{\mathrm{m}+i}+\sum_{j} \alpha_{\mathrm{m}+i}^{j} \overline{\mathrm{A}}_{j}, \\
\mathrm{KA}_{2 \mathrm{~m}+\mathrm{r}}=\alpha_{2 m+r}^{\mathrm{u}} \overline{\mathrm{A}}_{\mathrm{u}}, & \mathrm{r}=1, \ldots, \sigma, \quad u=1, \ldots, \mathrm{n}+1 .
\end{array}\right\}
$$

Although restrictions for $n$ are different in [4] and in the present paper ( $n \geq 4 m-1$ and $2 m-1 \leq n<3 m-1$ respectively), in the proof presented in [4] one needs to have $n \geq 2 m-1$ only.

Note that in the proof as consequences of (3.6) the author obtained the form (3.8) of the collineation $\mathrm{K}$, equalities

$$
\omega^{i}=\bar{\omega}^{i}
$$

giving developability of $\mathrm{c}$, and the following form for the 1-form $\theta_{1}$ in $(3.6)$ :

$$
\theta_{1}=\sum_{i}\left(-\tau_{i}^{i}+\alpha_{m+i}^{i} \rho_{i}^{-1} \omega^{i}\right) \text {. }
$$

In (3.10) and what follows we use the notation

$$
\tau_{\mathbf{u}}^{\mathrm{v}}=\bar{\omega}_{\mathrm{u}}^{\mathrm{v}}-\omega_{\mathrm{u}}^{\mathrm{v}}
$$

In addition, in what follows we will need the differential extension of equation (3.9) that has the following form:

$$
\tau_{m+i}^{m+i}-\tau_{i}^{i}=t_{i} \omega^{i}
$$

In the case of a projective deformation of second order one obtains from (3.3) conditions (3.6) and

$$
K d^{2}[A]=d^{2}[\bar{A}]+2 \theta_{1} d[\bar{A}]+\theta_{2}[\bar{A}]
$$

Differentiation of $(3.7)$ gives 


$$
\begin{aligned}
d^{2}[A] & =\sum_{i}\left[d \omega_{i}^{i}+\left(\omega_{i}^{i}\right)^{2}+\omega^{i} \omega_{m+i}^{i}\right][A]+\sum_{i}\left[d \omega^{i}+\omega^{i}\left(2 \sum_{j} \omega_{j}^{j}-\omega_{i}^{i}+\omega_{m+i}^{m+1}\right)\right]\left[A_{m+i}^{i}\right] \\
& +\underset{i, j}{ } \sum_{(i \neq j)}\left\{\left[c_{i}^{j}\left(\omega^{i}\right)^{2}-b_{i}^{j}\left(\omega^{j}\right)^{2}\right]\left[A_{m+j}^{i}\right]+\omega^{i} \omega^{j}\left[A_{m+i, m+j}^{i}, j\right\} .\right. \\
& \left.+a_{i}^{2 m+r}\left(\omega^{i}\right)^{2}\left[A_{2 m+r}^{i}\right]\right) .
\end{aligned}
$$

Note that equation (3.14) is slightly different from similar equation in the above mentioned paper [4] because of different restrictions for $n$ and different choice of moving frames.

THEOREM 2。 A correspondence $\mathrm{C}: \mathrm{L} \rightarrow \overline{\mathrm{L}}$ is a projective deformation of second order if and only if there exist functions $\rho_{i}$ such that the relative invariants $b_{i}^{j}$ and $\bar{b}_{i}^{j}$ of $\mathrm{L}$ and $\overline{\mathrm{L}}$ satisfy equation

$$
\rho_{i} \bar{b}_{i}^{j}=\rho_{j} b_{i}^{j}
$$

A collineation $K$ realizing such a deformation $C$ is determined by (2.8) where $\rho_{i}$ and $\alpha_{u}^{v}$ satisfy equations:

$$
\left.\begin{array}{l}
\rho_{1} \cdots \rho_{m}=1, \quad \rho_{i} c_{i}^{-k}-\rho_{k} c_{i}^{k}=a_{i}^{2 m+s} \alpha_{2 m+s}^{m+k}, \\
\rho_{i} t_{i}=a_{i}^{2 m+s} \alpha_{2 m+s}^{m+i}-2 \alpha_{m+i}^{i}
\end{array}\right\}
$$

Proof of Theorem 2 is computational and follows proof of a similar theorem in [4] where one should use (3.13) and (3.14).

Note also that in [4] the author proved that if $C: L \rightarrow \bar{L}$ is a projective deformation of second order, then the following identities hold:

$$
\begin{aligned}
& \rho_{i} \bar{b}_{i j}^{j}=\rho_{j} b_{i j}^{j}-b_{i}^{j}\left(\rho_{j} t_{j}-\alpha_{m+j}^{j}\right), \\
& \rho_{i} \bar{a}_{i j}^{j}=\rho_{j} a_{i j}^{j}-\bar{b}_{i}^{j} \alpha_{m+i}^{i} .
\end{aligned}
$$

They can be obtained from (3.15) and (2.15)。

4. SINGULAR PROJECTIVE DEFORMATIONS OF T.F. PSEUDOCONGRUENCES.

A correspondence $C: L \rightarrow \bar{L}$ induces the correspondences $C_{i}:\left(A_{i}\right) \rightarrow\left(\bar{A}_{i}\right)$ of focal surfaces of $L$ and $\vec{L}$. Suppose that there exists a collineation $H$ such that

$$
\mathrm{Hd}^{\mathrm{s}} \mathrm{A}_{i}=\sum_{\ell=0}^{s}\left(\begin{array}{c}
\mathrm{h} \\
\ell
\end{array}\right) \phi_{i}^{\ell} \mathrm{d}^{\mathrm{h}-\ell-\bar{A}_{i}}, \quad s=0,1, \ldots, \mathrm{h},
$$

where $\phi_{i}^{\ell}$ are $\ell$-forms. In this case we will say that $C_{i}$ is a projective deformation of order $h$ between $\left(A_{i}\right)$ and $\left(\bar{A}_{i}\right)$. A second order projective deformation $C: L \rightarrow \bar{L}$ realized by the collineation $\mathrm{K}$ which is determined by $(3.8)$ is said to be weakly singular, singular, or a-strongly singular, $\alpha=3,4, \ldots$, if the correspondences $C_{1}$ induced by $\mathrm{C}$ are projective deformations of order one, two or $\alpha$ respectively realized by the same collineation $\mathrm{K}$.

THEOREM 3. A second order projective deformation $C: L \rightarrow \bar{L}$ is weakly singular.

PROOF. Suppose that C: $\mathrm{L} \rightarrow \overline{\mathrm{L}}$ is a projective deformation of second order, $i_{0}$ e. we have (3.8) and (3.15)-(3.18). It follows from $(2.6)$ and $(2.10)$ that

$$
\mathrm{dA}_{i}=\omega_{i}^{i} \mathrm{~A}_{i}+\sum_{j \neq i} b_{i}^{j} \omega^{j} A_{j}+\omega^{i} A_{m+1} \text {. }
$$

Using (3.8), one finds from (4.2) that

$$
\mathrm{KA}_{i}=\rho_{i} \overline{\mathrm{A}}_{i}, \quad \mathrm{KdA}_{i}=\rho_{i} \overline{\mathrm{dA}}_{i}+\left(-\rho_{i} \tau_{i}^{i}+\alpha_{\mathrm{m}+i^{i}} \omega^{1}\right) \overline{\mathrm{A}}_{i} .
$$


According to $(4.1)$, it means that $\mathrm{K}$ realizes a projective deformation of first order of $\left(A_{i}\right)$ and $\left(\bar{A}_{i}\right)$ for any $i$ and therefore the correspondence $C: L \rightarrow \bar{L}$ is weakly singular。 Note also that $(4.3)$ and $(4.1)$ show that

$$
\phi_{i}^{0}=\rho_{i}, \quad \phi_{i}^{1}=-\rho_{i} \tau_{i}^{i}+\alpha_{m+i}^{i} \omega^{i} \text { 。 }
$$

THEOREM 4。 A second order projective deformation $C: L \rightarrow \bar{L}$ is singular if and only if the following equations hold:

$$
\begin{aligned}
& a_{i}^{2 m+r} \alpha_{2 m+r}^{j}=a_{i i_{j}}^{j}-\bar{a}_{i i_{i}}^{j} \rho_{i}-c_{i}^{j} \alpha_{m+j}^{j}, \\
& \rho_{i} a_{i}^{2 m+r}=a_{i}^{2 m+s} \alpha_{2 m+s}^{2 m+r} .
\end{aligned}
$$

PROOF。 Suppose again that $\mathrm{C}: \mathrm{L} \rightarrow \overline{\mathrm{L}}$ is a second order projective deformation. Differentiating $(4.2)$ and using $(2.1),(2.7),(2.8),(2.11),(2.12),(2.15)$, and $(2.16)$, we get

$$
\begin{aligned}
d^{2} A_{i} & =\left\{d \omega_{i}^{i}+\left(\omega_{i}^{i}\right)^{2}+\omega^{i}\left[\omega_{m+1}^{i}+\sum_{j \neq i} b_{i}^{j} b_{j}^{i} \omega^{j}\right]\right\} A_{i}+\sum_{j \neq i}\left[b_{i}^{j} d \omega^{j}+b_{i}^{j} \omega^{j}\left(2 \omega_{i}^{i}+\omega_{m+j}^{m+j}-\omega_{j}^{j}\right)-a_{i i}^{j}\left(\omega^{i}\right)^{2}\right. \\
& \left.+\omega^{j}\left(2 \sum_{k \neq i, j} b_{i}^{k} b_{k}^{j} \omega^{k}+b_{i j}^{j} \omega^{j}+2 a_{i j}^{j} \omega^{i}\right)\right] A_{j}+\left[d \omega^{i}+\omega^{i}\left(\omega_{i}^{i}+\omega_{m+i}^{m+i}\right)\right] A_{m+i} \\
& +\sum_{j \neq i}\left[b_{i}^{j}\left(\omega^{j}\right)^{2}+c_{i}^{j}\left(\omega^{i}\right)^{2}\right] A_{m+j}+a_{i}^{2 m+r}\left(\omega^{i}\right)^{2} A_{2 m+r}
\end{aligned}
$$

Using $(3.11),(4.7),(\overline{4.7}),(3.8),(4.3),(\overline{4.2}),(4.5),(4.6),(3.12)$, and $(3.15)-(3.18)$, we obtain

$$
\begin{aligned}
K d^{2} A_{i}-\rho_{i} d^{2} \bar{A}_{i}-2 \phi_{i}^{1} \overline{d A}_{i}-\phi_{i}^{2} \bar{A}_{i} & =\left(\omega^{i}\right)^{2}\left[\sum_{k \neq i}\left(a_{i}^{2 m+r} \alpha_{2 m+r}^{k}+a_{i i}^{k} \rho_{i}-a_{i i}^{k} \rho_{k}+c_{i}^{k} \alpha_{m+k}^{k}\right) \bar{A}_{k}\right. \\
& \left.+\left(a_{i}^{2 m+s} \alpha_{2 m+s}^{2 m+r}-\rho_{i} \bar{a}_{i}^{2 m+r}\right) \bar{A}_{2 m+r}\right]
\end{aligned}
$$

where

$$
\phi_{i}^{2}=-\rho_{i}\left[d \tau_{i}^{i}-\left(\tau_{i}^{i}\right)^{2}+\omega^{i} \tau_{m+i}^{i}\right]+\alpha_{m+i}^{i}\left[d \omega^{i}+\omega^{i}\left(\omega_{m+i}^{m+i}-2 \tau_{i}^{i}-\omega_{i}^{i}\right)\right]+a_{i}^{2 m+r} \alpha_{2 m+r}^{i}\left(\omega^{i}\right)^{2} .
$$

Comparison of (4.1) for $s=2$ and (4.8) leads to equations (4.5) and (4.6)。 Q.E.D.

THEOREM 5. Suppose that $\mathrm{L}$ and $\overline{\mathrm{L}}$ are second class t.f. pseudocongruences of planes in projective spaces $\mathrm{P}^{\mathrm{n}}$ and $\overline{\mathrm{P}}^{\mathrm{n}}, 2 \mathrm{~m}-1 \leq \mathrm{n}<3 \mathrm{~m}-1$, and suppose that they are related by a second order projective deformation $\mathrm{C}: \mathrm{L} \rightarrow \overline{\mathrm{L}}$. The pseudocongruences $\mathrm{L}$ and $\overline{\mathrm{L}}$ are identical if and only if the deformation $\mathrm{C}$ is 3-strongly singular。

PROOF. Suppose again that $\mathrm{C}: \mathrm{L} \rightarrow \overline{\mathrm{L}}$ is a second order projective deformation between $L$ and $\bar{L}$. The deformation $C$ is 3-strongly singular if (4.1) holds for $s=1,2,3$ 。 We already showed that for $s=1$ equation ( 4.1 ) holds automatically and for $s=2$ it holds if and only if conditions (4.5) and (4.6) are satisfied.

For a 3-strongly singular deformation $C$ we additionally have

$$
K d^{3} A_{i}=\rho_{i} d^{3} \bar{A}_{i}+3 \phi_{i}^{1} d^{2} \bar{A}_{i}+3 \phi_{i}^{2} \overline{d A}_{i}+\phi_{i}^{3} \bar{A}_{i}
$$

where $\phi_{i}^{1}$ and $\phi_{i}^{2}$ are 1 - and 2-forms determined by (4.4) and (4.9) and $\phi_{i}^{3}$ is a 3-form. Differentiating (4.7) and using $(2.1),(2.7),(2.8),(2.11)$, and $(2.15)-(2.21)$, we obtain

$$
d^{3} A_{i}=(\cdot) A_{i}+\sum_{j}\left(\psi_{i}^{j} A_{j}+\psi_{i}^{m+j} A_{m+j}\right)+\psi_{i}^{2 m+r} A_{2 m+r}
$$


where for $j \neq i$ :

$$
\left.\begin{array}{l}
\psi_{i}^{j}=2 \omega^{i} \omega^{j}\left(a_{i j j}^{j} \omega^{j}-a_{i i j}^{j} \omega^{i}\right)+\left(\omega^{j}\right)^{2}\left(b_{i j j}^{j} \omega^{j}+a_{i j j}^{j} \omega^{i}\right)-\left(\omega^{i}\right)^{2}\left(a_{i i j}^{j} \omega^{j}-c_{i i j}^{j} \omega^{j}\right)+\beta_{i}^{j}, \\
\psi_{i}^{m+i}=\omega_{m+i}^{i} \omega^{i}+\beta_{i}^{m+i}, \\
\psi_{i}^{m+j}=c_{i i}^{j}\left(\omega^{i}\right)^{3}+\beta_{i}^{m+j}, \\
\psi_{i}^{2 m+r}=a_{i i}^{2 m+r}\left(\omega^{i}\right)^{3}-a_{i}^{2 m+r} \omega_{2 m+r}^{2 m+r}\left(\omega^{i}\right)^{2}+\beta_{i}^{2 m+r} .
\end{array}\right\}
$$

In (4.12) we denoted by $\beta_{u}^{V}$ 2-forms which produce terms vanishing on our final step because of conditions imposed by a second order singular projective deformation $C$ and the first conditions following from (4.11) which we are going to obtain on our final step.

Before making the final step of our proof let us simplify at first a collineation K. By a suitable choice of local frames we obtain

$$
\mathrm{KA}_{\mathrm{u}}=\overline{\mathrm{A}}_{\mathrm{u}} \text {. }
$$

Equation (4.13) means that

$$
\alpha_{\mathrm{u}}^{\mathrm{v}}=\delta_{\mathrm{u}}^{\mathrm{v}}
$$

It follows from $(4.14),(3.9)-(3.11),(3.15)-(3.18),(4.5),(4.6),(2.5),(2.10),(2.7)$, $(2.8),(2.11),(2.12),(2.16),(4.4)$, and $(4.9)$ that

$$
\begin{aligned}
& b_{i}^{j}=\vec{b}_{i}^{j}, \quad c_{i}^{j}=\bar{c}_{i}^{j}, \quad a_{i j}^{j}=\bar{a}_{i j}^{j}, \quad b_{i j}^{j}=\bar{b}_{i j}^{j}, a_{i i}^{j}=a_{i i}^{j}, a_{i}^{2 m+r}=a_{i}^{2 m+r} \\
& \tau_{i}^{m+i}=\tau_{i}^{j}=\tau_{i}^{m+j}=\tau_{i}^{2 m+r}=\tau_{m+i}^{j}=\tau_{m+i}^{m+j}=\tau_{m+i}^{2 m+r}=0, j \neq i \\
& \phi_{i}^{1}=-\tau_{i}^{i}, \phi_{i}^{2}=-d \tau_{i}^{i}+\left(\tau_{i}^{i}\right)^{2}-\tau_{m+i}^{i} \omega^{i}
\end{aligned}
$$

In addition, equations (3.16), (3.12), and equation (4.5) of [4] imply

$$
\tau_{i}^{i}=\tau_{j}^{j}=\tau_{m+i}^{m+i}=\tau_{m+j}^{m+j}, \quad j \neq i \text {. }
$$

It follows from $(2.4)$ and $(4.18)$ that

$$
2 m \tau_{i}^{i}+\tau_{2 m+r}^{2 m+r}=0 \quad \text { (no summation). }
$$

Applying (4.13) to $(4.11)$ and using $(\overline{4.13}),(\overline{4.7}),(\overline{4.2}),(4.17)$, and conditions of singularity of $\mathrm{C}$, we get

$$
K d^{3} A_{i}-\left(d^{3} \bar{A}_{i}+3 \phi_{i}^{1} d^{2} \bar{A}_{i}+3 \phi_{i}^{2} \overline{d A}_{i}+\phi_{i}^{3} \bar{A}_{i}\right)=\sum_{j \neq i} \Omega_{i}^{j} \bar{A}_{j}+\sum_{j} \Omega_{i}^{m+j} \bar{A}_{m+j}+\Omega_{i}^{2 m+r} \bar{A}_{2 m+r}
$$

where for $j \neq i$ :

$$
\begin{aligned}
& \Omega_{i}^{j}=\left(b_{i j j}^{j}-b_{i j j}^{j}\right)\left(\omega^{j}\right)^{3}+\left(c_{i i j}^{j}-c_{i i j}^{j}\right)\left(\omega^{i}\right)^{3}+3 \omega^{i} \omega^{j}\left[\left(a_{i j j}^{j}-a_{i j j}^{j}\right) \omega^{j}\right. \\
& \Omega_{i}^{m+i}=2 \tau_{m+i}^{i}\left(\omega^{i}\right)^{2}+\gamma_{i}^{m+1}, \\
& \left.\left.a_{i i j}^{j+j}-a_{i i j}^{j}\right) \omega^{i}\right]+\gamma_{i}^{j}, \\
& \Omega_{i}^{m}=\left(c_{i i}^{j}-\bar{c}_{i i}^{j}\right)\left(\omega^{i}\right)^{3}, \\
& \Omega_{i}^{2 m+r}=a_{i}^{2 m+r} \tau_{2 m+r}^{2 m+r}\left(\omega^{i}\right)^{2}+\left(a_{i i}^{2 m+r}-a_{i i}^{2 m+r}\right)\left(\omega^{i}\right)^{3}, \\
& \text { and } \gamma_{u} \text { in }(4.21) \text { are 3-forms vanishing on our final step. }
\end{aligned}
$$


Comparison of $(4.11)$ and $(4.20)$ leads us to the following conclusions:

i) First of all, we get from the comparison that

$$
\tau_{2 m+r}^{2 m+r}=\left(a_{i i}^{2 m+r}-a_{i i}^{2 m+r}\right)\left(a_{i}^{2 m+r}\right)^{-1} \omega^{i} \quad \text { (no summation). }
$$

Since $\omega^{i}$ are linearly independent, it implies

$$
\begin{aligned}
& \tau_{2 m+r}^{2 m+r}=0 \quad \text { (no summation), } \\
& a_{i i}^{2 m+r}=a_{i i}^{2 m+r} .
\end{aligned}
$$

Equations $(4.19),(4.22)$, and $(4.18)$ give

$$
\tau_{i}^{i}=\tau_{m+i}^{m+i}=0 \text {. }
$$

It follows from $(4.23),(2.18)$, and $(\overline{2.18})$ that

$$
a_{i}^{2 m+s} \tau_{2 m+s}^{2 m+r}=0
$$

ii) Second of all, the comparison gives

$$
c_{i i}^{j}=c_{i i}^{j}
$$

Equations $(4.26),(4.15),(2.17)$, and $\overline{(2.17)}$ leads to

$$
a_{i}^{2 m+s} \tau_{2 m+s}^{j}=0
$$

iii) Further, we obtain from the comparison that

$$
\tau_{m+i}^{i}=0
$$

Note that $\gamma_{i}^{m+i}=0$ by means of $(4.24)$ 。

i.v) Finally, the comparison gives

$$
a_{i i j}^{j}=\bar{a}_{i i j}^{j}, \quad a_{i j j}^{j}=\bar{a}_{i j j}^{j}, \quad b_{i j j}^{j}=\bar{b}_{i j j}^{j}, \quad c_{i i j}^{j}=\bar{c}_{i i j}^{j} .
$$

Note that $\gamma_{i}^{j}=0$ by means of $(4.24)$ and other conditions which were previously obtained. It follows from $(4.29),(4.15),(2.21)$, and $\overline{(2.21)}$ that

$$
a_{i}^{2 m+s} \tau_{2 m+s}^{j}=0 \text { 。 }
$$

Lquality (2.14) means that the rank of the matrix of coefficients of each of the linear homogeneous systems $(4.25),(4.27)$, and $(4.30)$ is maximal and equal to the number of unknowns. Therefore, these systems lead to

$$
\tau_{2 m+r}^{j}=\tau_{2 m+r}^{m+j}=\tau_{2 m+r}^{2 m+s}=0
$$

Equations $(4.16),(4.22),(4.24),(4.28)$, and $(4.31)$ show that all forms $\tau_{u}^{v}=0$ 。 Therefore, the pseudocongruences $\mathrm{L}$ and $\overline{\mathrm{L}}$ are identical. The converse statement is trivial。

Q.E.D。

\section{REFERENCES}

1. SVEC, A。 Projective Differential Geometry of Line Congruences, Czechoslovak Academy of Sciences, Prague, 1965.

2. GOLDBERG, L. G. (Pikuleva, L. G.) A projective deformation of totally focal pseudocongruences of planes in multidimensional projective spaces (Russian), Kalinin Gos. Ped. Inst. Uchen。Zap., 74 (1970) 111-123.

3. GOLDBERG, L. G. (Pikuleva, L. G。) A singular projective deformation of totally focal pseudocongruences of planes in multidimensional projective spaces (Russian), Akad. Nauk Armyan SSR Dok1。60 (1975), No. 5, 257-262. 
4. GOLDBERG, L. G. The third order singular projective deformations of totally focal pseudocongruences of planes in multidimensional projective spaces, Atti Accad。 Peloritana Pericolanti 62 (1984), 45-72 (1986).

5. KREIZLIK, J. Deformations of plane pseudocongruences with projective connection, Czech. Math. J. 21 (96) (1971) 213-233.

6. KREIZLIK, J. Contribution to the theory of pseudocongruences with projective connection, Arch. Math. (Brno) 17 (1981), no。1,31-41。

7. GEIDELMAN, R。 Mo A theory of pseudocongruences and congruences of planes of multidimensional hyperbolic space and congruences of spheres of multidimensional conformal space (Russian), Mat。Sb。 36 (78) (1955), No。2, 209-232. 


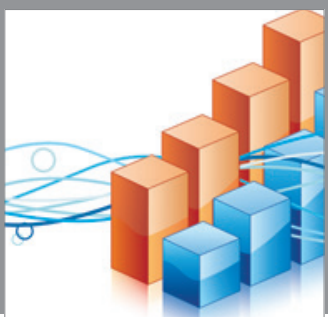

Advances in

Operations Research

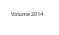

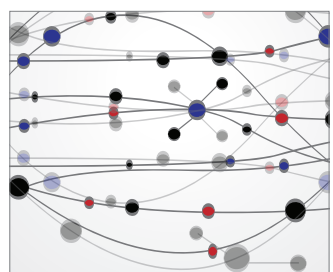

\section{The Scientific} World Journal
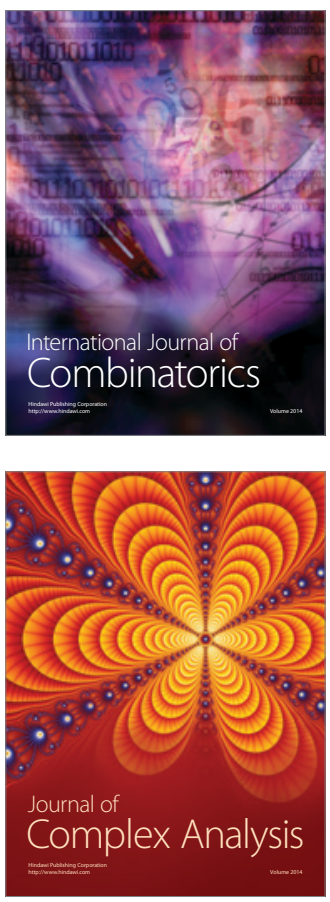

International Journal of

Mathematics and

Mathematical

Sciences
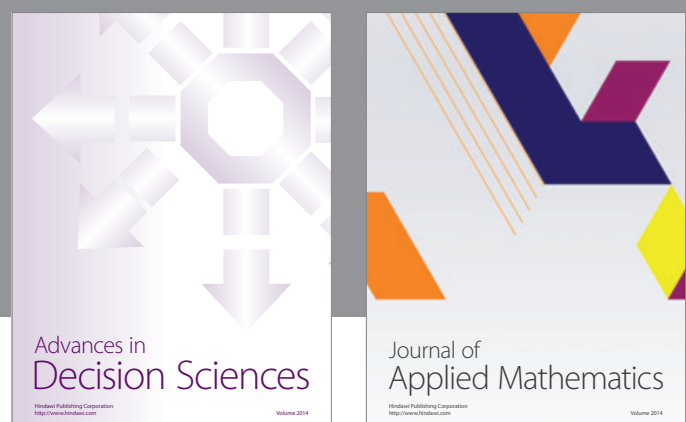

Journal of

Applied Mathematics
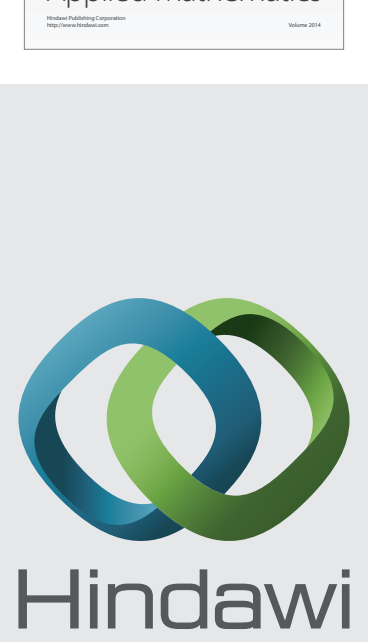

Submit your manuscripts at http://www.hindawi.com
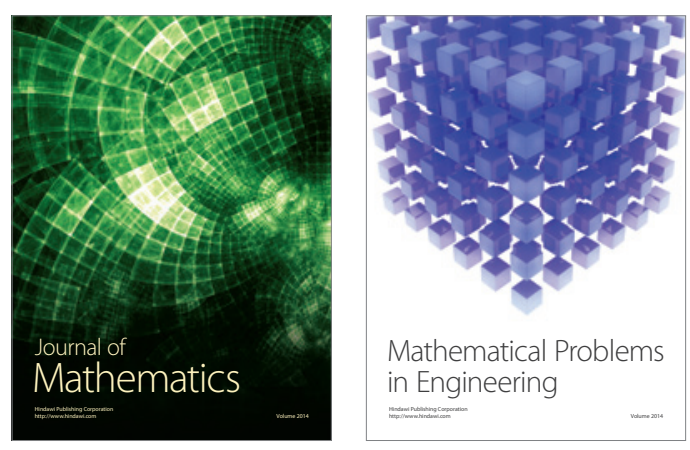

Mathematical Problems in Engineering
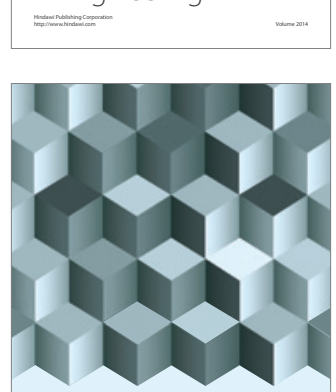

Journal of

Function Spaces
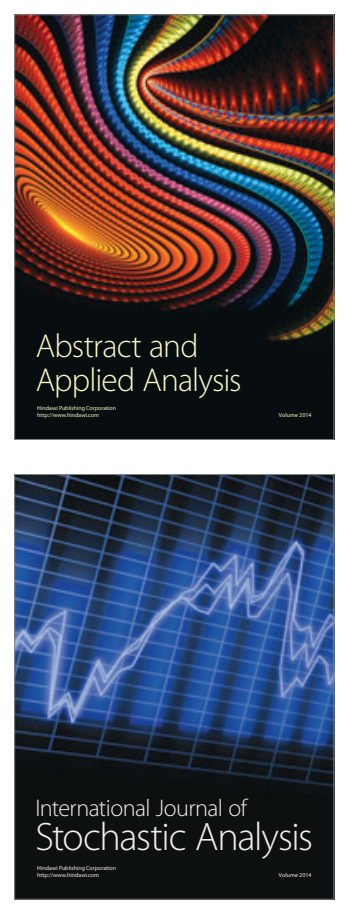

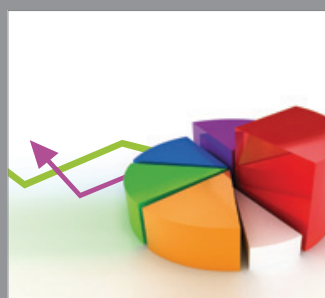

ournal of

Probability and Statistics

Promensencen
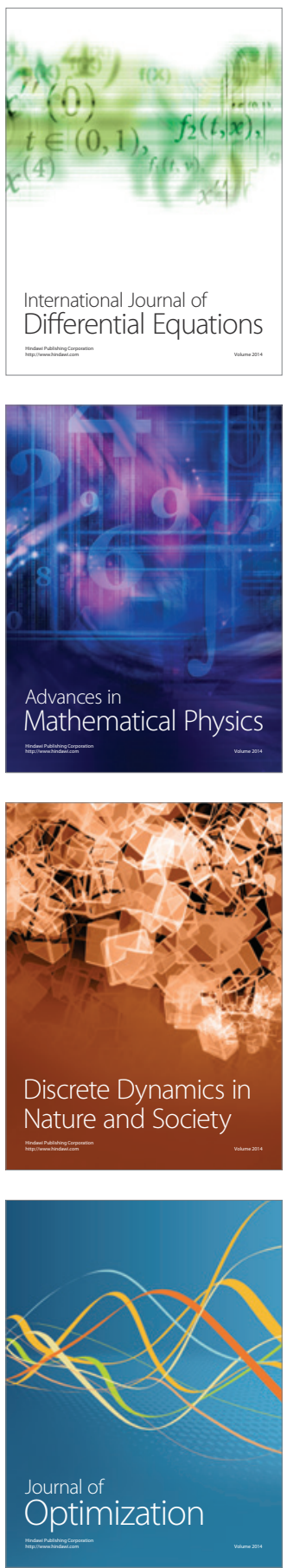